\title{
Association Between Severity of Peripheral Artery Disease Angio CT- derived Coronary Syntax Score in Patients with Critical Limb Ischemia
}

\author{
Emese Rapolti¹, Theodora Benedek¹, István Kovács¹, Szilamér Korodi¹, Zsuzsanna Suciu1, \\ Sebastian Condrea², Edvin Benedek², András Mester², Imre Benedek \\ ${ }^{1}$ Clinic of Cardiology, University of Medicine and Pharmacy, Tîrgu Mureș, Romania \\ 2 Center of Advanced Research in Multimodality Cardiac Imaging, Cardio Med Medical Center, Tîrgu Mureș, Romania
}

\section{CORRESPONDENCE}

\section{András Mester}

76, 22 decembrie 1989 St

540124 Tîrgu Mureș, Romania

Tel: 0265217333

Email: andras.mester@yahoo.com

\section{ARTICLE HISTORY}

Received: 2 April, 2016

Accepted: 18 May, 2016

Emese Rapolti • 38 Gheorghe Marinescu St, 540139 Tîrgu Mureș, Romania, Tel: +40 265215551 Email: rapoltie@yahoo.com

Theodora Benedek • 38 Gheorghe Marinescu St, 540139 Tîrgu Mureș, Romania, Tel: +40 265215551 Email: theodora.benedek@gmail.com

István Kovács • 38 Gheorghe Marinescu St, 540139 Tîrgu Mureș, Romania, Tel: +40 265215551 Email:kov_istvan@yahoo.com

Szilamér Korodi • 38 Gheorghe Marinescu St, 540139 Tîrgu Mureș, Romania, Tel: +40 265215551 Email: szilamerkorodi@yahoo.com

Zsuzsanna Suciu • 38 Gheorghe Marinescu St, 540139 Tîrgu Mures, Romania, Tel: +40 265215551 Email: szoke_zsuzsanna@yahoo.com

Sebastian Condrea • 76, 22 Decembrie 1989 St 540124 Tîrgu Mures, Romania, Tel: +40 265217333 Email: sebastian.condrea@yahoo.com

Edvin Benedek • 76, 22 Decembrie 1989 St, 540124 Tîrgu Mures, Romania, Tel: +40 265217333 Email: edvinke87@gmail.com

Imre Benedek • 38 Gheorghe Marinescu St, 540139 Tîrgu Mures, Romania, Tel: +40 265215551

Email: imrebenedek@yahoo.com

\begin{abstract}
Introduction: Peripheral artery disease, a frequent consequence of atherosclerosis, is usually associated with concomitant ischaemic coronary artery disease and with a high rate of cardiovascular mortality. Material and methods: The study population consisted of 24 patients, admitted to our clinic with peripheral artery disease, 10 of them with critical limb ischaemia. In all cases, cardiovascular risk factors, left ventricular dysfunction and ejection fraction were analyzed. Peripheral Multislice Angio CT examination was used to determine the TASC class and to assess the peripheral arterial lesions. The Coronary Calcium Score and the Syntax Score were determined with angio CT of the coronary arteries. Results: Patients were between 39 and 84 years of age, and 54\% were in Fontaine class 2B, 21\% in Fontaine class 3 and $25 \%$ in Fontaine class $4.12 .5 \%$ of patients presented TASC class A, 33.3\% TASC class B, and 54.2\% TASC class C. Coronary Calcium Scores were between 0 and 100 in $16.6 \%$ of patients, between 100 and 400 in $41.8 \%$, and $>400$ in $41.6 \%$ of patients. The SYNTAX Score was $<22$ in $54 \%$ of patients, between 22 and 32 in 37.5\%, and $>32$ in $8.5 \%$ of patients. A significant correlation was found between the Coronary Calcium Score and the SYNTAX Score $(r=0.82, p$ $=0.03$ ). The Syntax Score was $22.43 \pm 3.2$ in TASC A patients, $26.2 \pm 5.4$ in TASC B patients, $32.1 \pm 2.3$ in TASC $C$ patients $(p=0.005)$. Conclusions: The severity of coronary artery disease characterized by the Syntax Score, by the presence of left main stenosis and segmental left ventricular hypokinesis presents significant correlation with the severity of peripheral artery disease, characterized by the TASC classification.
\end{abstract}

Keywords: peripheral arterial disease, TASC class, Syntax Score

\section{INTRODUCTION}

Peripheral artery disease and coronary artery disease are the most important and severe consequences of the systemic atherosclerotic process. Peripheral artery disease is present in more than 200 million people worldwide and the 


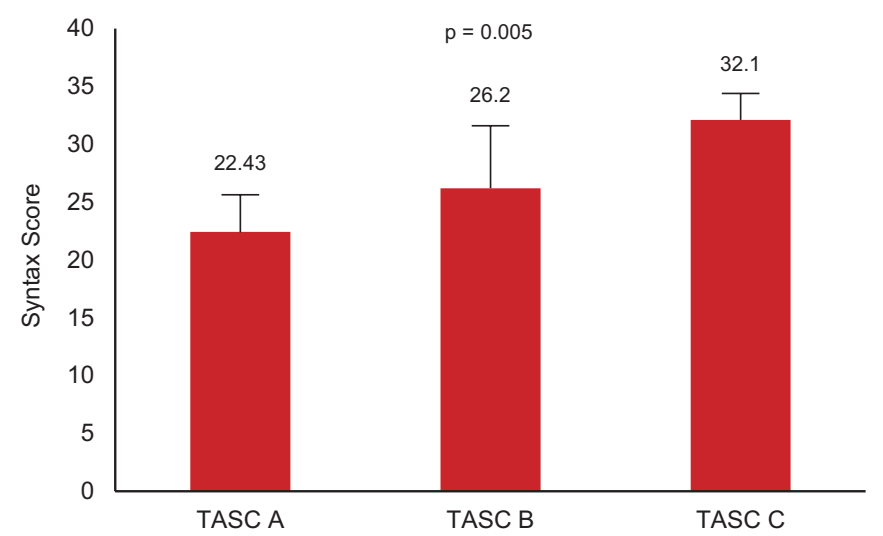

FIGURE 1. Correlation between the Syntax Score and TASC class

prevalence of peripheral artery disease increases after the age of 50 years. $^{1}$ Peripheral artery disease in untreated patients leads to critical limb ischaemia, and this leads in most of the cases to amputation of the affected lower limb, increasing cardiovascular and all-cause mortality as well. In adults, critical limb ischaemia appears in $12 \%$ of cases and is more frequent in patients at older age. In the population group above 70 years of age, the prevalence is $20 \%{ }^{2}$

High blood pressure, smoking, dyslipidemia and the presence of diabetes facilitate the formation of atheromatous plaques not only in the coronary arteries, but also in the peripheral arteries. In patients with severe peripheral artery disease we can find associated coronary artery disease. To reduce the rate of mortality and to optimize the quality of life of our patients, early diagnosis and initiation of proper treatment addressed to both peripheral artery disease and coronary artery disease are required.

Advanced non-invasive vascular imaging of the peripheral arteries and coronary arteries can be provided by Multislice Angio Computer Tomography, a technique that offers high resolution and high degree of accuracy in a short period of examination.

\section{MATERIAL AND METHODS}

Our study population consists of 24 patients, who were admitted at the Cardio Med Medical and Research Center with peripheral artery disease between August 1 1st, 2015 and December 31, 2015. In all patients, peripheral Doppler examination, ECG, echocardiography, evaluation of cardiovascular risk factors, angio $\mathrm{CT}$ of the lower extremities and angio $\mathrm{CT}$ of the coronary arteries with calculation of the Syntax Score and Calcium Score were performed.

The study has been carried out in accordance with the code of ethics of the World Medical Association's Declara-

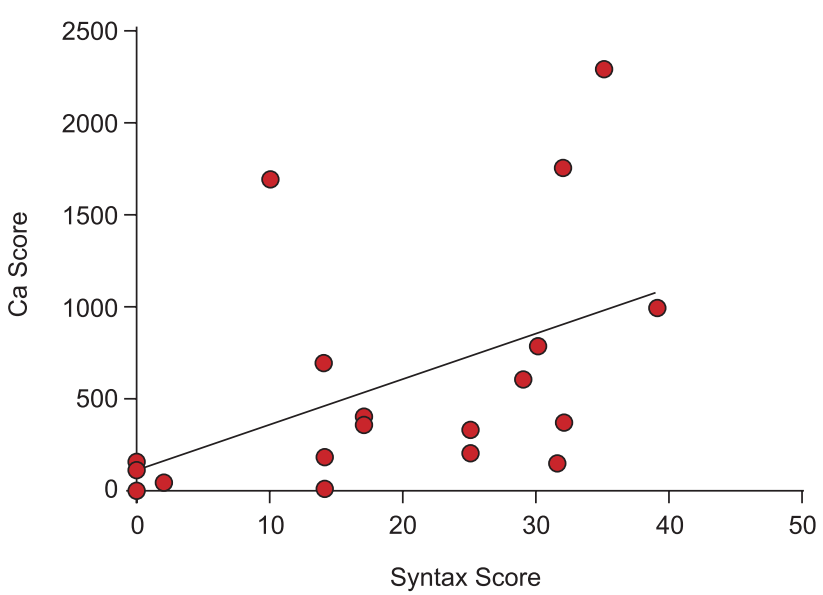

FIGURE 2. Correlation between the Coronary Calcium Score and the Syntax Score.

tion of Helsinki. All patients gave written informed consent, and the study protocol was approved by the ethics committee of the Cardio Med Medical Center, the center where the study was conducted.

\section{Statistical analysis}

The statistical analysis was performed using the GraphPad Prism 7 software. Categorical data were compared using the Chi-square and Fisher's exact tests. The unpaired t test was used to test for differences between the two groups, regarding the continuous variables.

\section{RESULTS}

Our patients were between the ages of 39 and $84,83 \%$ male, $17 \%$ female patients, who at admission presented intermittent claudication and an ankle brachial index less than 0.7 . Forty-two percent of our patients presented critical limb ischaemia. At the evaluation of the cardiovascular risk factors we found that $88 \%$ of the patients presented hypertension, $12 \%$ had normal blood pressure and $53 \%$ of them presented high cholesterol level. Renal dysfunction was present in $32 \%$ of patients. Forty-five percent of patients were smokers and $54.3 \%$ were diagnosed with diabetes.

Echocardiography showed a reduced ejection fraction $(<40 \%)$ in $41 \%$ of patients, and segmental left ventricular hypokinesis in $79 \%$ of patients, especially in those with class TASC B and TASC C. Fifty-four percent of our patients presented Rutherford class 3, $21 \%$ presented Rutherford class 4 , and $25 \%$ of patients were admitted to our clinic with Rutherford class 5 . We determined the TASC 


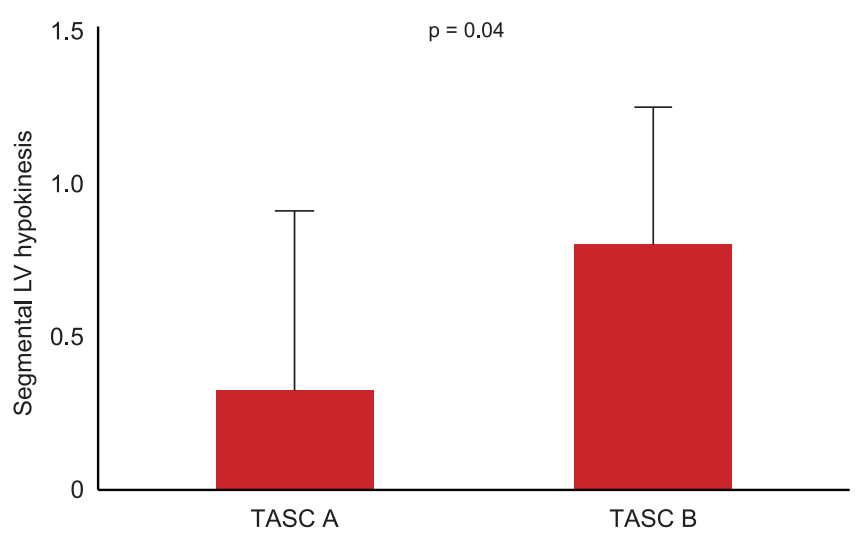

FIGURE 3. Correlation between segmental left ventricular (LV) hypokinesia and TASC class in patients with peripheral artery disease.

class with Multislice Angio CT 64: the majority of the patients, $54.2 \%$ were in class TASC C, $33.3 \%$ of patients in class TASC B, and we had only a few patients, $12.5 \%$ in class TASC A.

Coronary Calcium Score, Syntax Score, and coronary lesions were determined based on coronary angio CT. $87.5 \%$ of patients presented severe coronary lesions with indication for invasive angiography and percutanous coronary angioplasty. We have found a low calcium score, between 0 and 100 in $16.6 \%$ of patients, an intermediate calcium score, between 100 and 400 in $41.8 \%$ of patients, and a high calcium score in $41.6 \%$ of patients. Significant left main stenosis was present in $59 \%$ of patients, severe tricoronary lesions in $35 \%$ of patients, bicoronary lesions in $23 \%$ of patients, one significant coronary lesion with indication for coronary angioplasty in $28 \%$ of patients. Only $14 \%$ of patients presented no significant coronary lesions, but atheromatous plaques were present in these patients' coronary arteries too. Significant left main stenosis, severe tricoronary and bicoronary lesions were present especially in patients with critical limb ischaemia and class TASC C. Fifty-four percent of patients presented a Syntax Score between 0 and 22, 37.5\% between 22 and 32, while the Syntax Score was higher than 32 in $8.5 \%$ of patients.

In this study we assessed the correlation between the severity of arterial disease and scores expressing the severity of coronary atherosclerosis, such as the Syntax Score, in patients with critical limb ischaemia. We found a significant correlation between the Syntax Score and TASC classification ( $p=0.001$, Figure 1$)$, between the presence of left main stenosis and Rutherford stadium ( $\mathrm{p}=0.03$ ), and a good correlation between the Coronary Calcium Score and the Syntax Score $(\mathrm{p}=0.03, \mathrm{r}=0.82$, Figure 2$)$. The Syntax Score was $22.43 \pm 3.2$ in TASC A patients, $26.2 \pm$ 5.4 in TASC B patients, $32.1 \pm 2.3$ in TASC C patients ( $\mathrm{p}$ $=0.005)$.

The presence of segmental left ventricular hypokinesia presented a good correlation with the TASC class, which means that the severity of peripheral artery disease is increasing in parallel with the deterioration of the left ventricular function produced by ischaemic coronary artery disease ( $p=0.04$, Figure 3 ).

\section{DISCUSSION}

This study shows that the majority of patients with peripheral artery disease present coronary artery disease too. Significant coronary lesions were present in $87.5 \%$ of our patients, although more than half of them were free of any anginal symptoms. Occlusion of the bilateral superficial
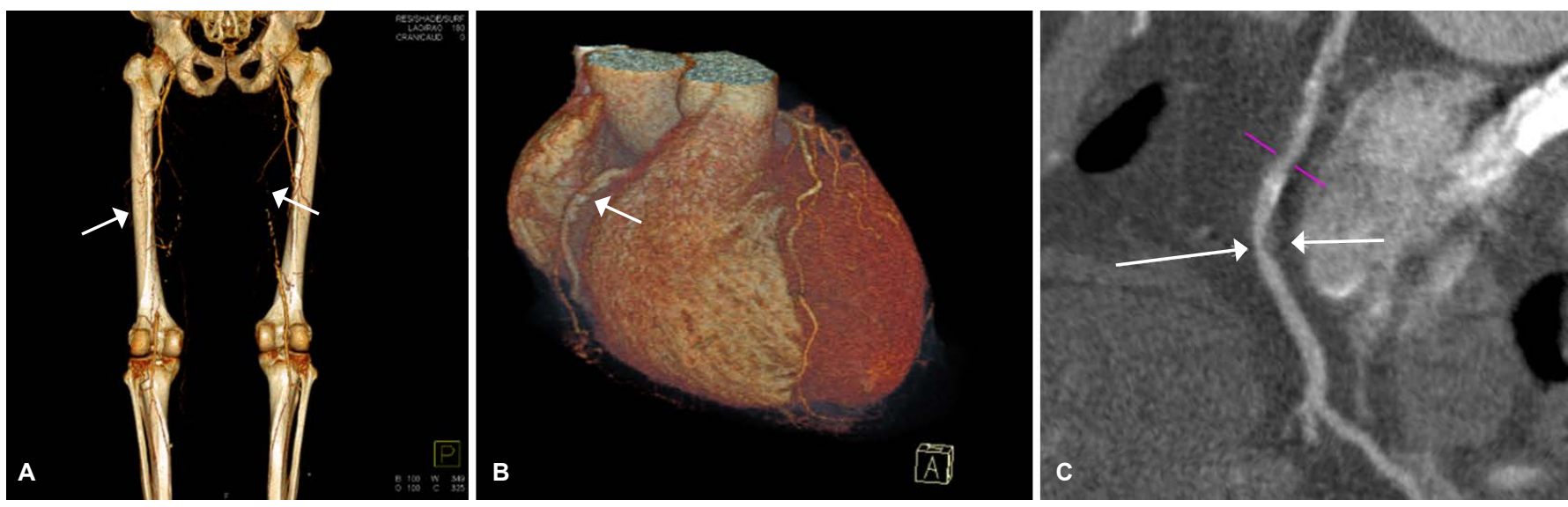

FIGURE 4. Angio CT of the lower limbs in a patient with peripheral artery disease, showing bilateral occlusion of the femoral arteries (A), associated with Angio CT examination of the coronary arteries indicating calcifications in the righ coronary artery (B) and a stenosis in the middle segment of the right coronary artery (C). 
femoral arteries can be associated with the presence of calcified plaques and significant stenosis of the right coronary artery (Figure 4). This study proves that the screening of coronary artery disease in patients diagnosed with peripheral artery disease is essential in order to prevent major cardiac events.

There are similar studies published in the literature revealing the association between peripheral artery disease and coronary artery disease. A study on 182 patients showed that coronary artery disease was two times more frequent in patients who had peripheral artery disease, than in patients who had no peripheral artery disease. In this study coronary artery disease was present in $46.88 \%$ of patients who were diagnosed with peripheral artery disease. ${ }^{3}$

Another study which investigated the progression of coronary atherosclerosis in 3479 patients diagnosed with coronary artery disease, showed that the progression of the atheroma volume was greater in patients who had associated peripheral artery disease. Patients who had concomitant coronary artery disease and peripheral artery disease experienced more often major cardiac events. ${ }^{4}$

However, the inflammatory process which is present at the claudicant limb, may aggravate the progression of atherosclerosis in the coronary arteries. Patients who present marked inflammatory status exhibit more often an associated severe multivascular coronary artery disease. ${ }^{5}$

\section{CONCLUSIONS}

Patients with peripheral artery disease present concomitant coronary artery disease, which is proven by the positive correlation found between TASC classification and the Syntax Score. There is a significant correlation between the severity of peripheral artery disease character- ized by Rutherford stadialization and the presence of significant left main stenosis. The presence of segmental left ventricular hypokinesis also presents a good correlation with the TASC classification, which shows that there is an association between ischaemic coronary artery disease and peripheral artery disease.

This study proves that the atherosclerotic process shows similar severity in the coronary arteries and the peripheral arteries. Major cardiac events in patients suffering from peripheral artery disease, including critical limb ischaemia, can be prevented by screening and treating the associated coronary artery disease.

\section{CONFLICT OF INTEREST}

Nothing to declare.

\section{ACKNOWLEDGEMENT}

This research was supported by the Cardio Med Medical Center, project no. CAM/2015/RD/36.

\section{REFERENCES}

1. Criqui $\mathrm{MH}$, Aboyans $\mathrm{V}$. Epidemiology of peripheral artery disease Circulation Research. 2015;116:1509-1526.

2. Davies GM. Criticial Limb Ischemia: Epidemiology. Methodist Debakey Cardiovasc J. 2012:8(4):10-14

3. Sarangi S, Srikant B, Rao DV, Joshi L, Usha G. Correlation between peripheral arterial disease and coronary artery disease using ankle brachial index-a study in Indian population. Indian Heart J. 2012;64(1):2-6.

4. Hussein AA, Uno K, Wolski K, et al. Peripheral Arterial Disease and Progression of Coronary Atherosclerosis. J Am Coll Cardiol 2011;57(10):1220-1225.

5. Brevetti G1, Piscione F, Schiano V, Galasso G, Scopacasa F, Chiariello M. Concomitant coronary and peripheral arterial disease: Relationship between the inflammatory status of the affected limb and the severity of coronary artery disease. J Vasc Surg. 2009 Jun;49(6):1465-71. 